\title{
Characteristics of admissions considered inappropriate by junior psychiatrists
}

\author{
Peter Y. Elwood
}

\begin{abstract}
Aims and method The aim of the study was to identify medical and socio-demographic characteristics of admissions considered inappropriate by psychiatrists. Appropriateness of admission was assessed by questionnaire. Medical and socio-demographic characteristics of each admission were obtained by case note review.

Results Twenty-five per cent of admissions were considered inappropriate. These patients showed high levels of adverse socio-demographic characteristics and commonly were diagnosed as personality disordered or as substance misusers. Junior doctors commonly admitted patients despite considering admission inappropriate.
\end{abstract}

Clinical implications Regular audit of the admission, process should be encouraged.

Admission to a psychiatric hospital is a complex process mediated by a number of behavioural, social and medical variables. Goldberg \& Huxley (1980) proposed a model demonstrating how the patient, general practitioner and psychiatrist act as a series of filters in the presentation and subsequent treatment of mental illness.

Concerning the final filter by which the patient is admitted to a psychiatric hospital, adverse socio-demographic characteristics of the patient such as unemployment, divorce and medical characteristics including a past history of admission, parasuicide or psychosis may positively predict admission (Stein \& Test, 1980; Hoult, 1986; Kent \& Yellowlees, 1994). Attitudes and experience of the admitting doctor also influence the admission process (Meyerson et al, 1979; Flannigan et al, 1994).

Studies of the appropriateness of admission have mostly concerned general hospitals with psychiatric admission receiving little attention. However, Beck et al (1997) surveyed 481 psychiatric admissions over six months in Nottingham and identified alternatives to hospitalisation for $29 \%$ of admissions, while Strumwasser et al (1991) analysed 539 admissions in Michigan, and suggested that $40 \%$ of bed days utilised by general psychiatric patients and $60 \%$ utilised by patients with a diagnosis of substance misuse were occupied inappropriately.

It is important to identify and avoid inappropriate admission. Much has been written from the patient's perspective regarding stigmatisation (Scambler, 1991), medicalisation of problems and diminished coping strategies (Hoult, 1986) and ultimately institutionalisation (Goffman, 1961). Less attention has been paid to the cost of inappropriate admission. Hicks (1994) emphasises the importance of avoiding overuse of health services (patients being offered inappropriate health care) and underuse of services (patients not being offered appropriate health care). Both are related in that if beds are filled inappropriately, then those truly in need of admission miss out, particularly in a climate of bed storages. Both Flannigan et al (1994) and Beck et al (1997) suggest that some psychiatric in-patients could be treated in less intensively staffed community alternatives. In the most recent comparison of costs of day versus inpatient treatment, Creed et al (1997) showed day hospital treatment to be a viable cheaper proposition for a number of potential admissions, many of them people with schizophrenia.

The aims of this study are: first, to quantify the number of admissions considered inappropriate by the admitting psychiatrist: second, to establish the socio-demographic and medical characteristics of these admissions and to compare them against those of patients whose admission was considered appropriate in a case-control study: and finally, to compare the length of stay of admissions considered inappropriate and appropriate.

\section{The study}

The study was performed in York, a psychiatric hospital of 80 acute beds covered by seven consultants and 13 trainees. All patients admitted over a four-month period (April-July 1996) were included, the only exclusions being 
those whose admission the author was involved in.

On admission the admitting psychiatrist recorded his grade and the time of the admission on a questionnaire. He allocated the patient to an ICD-10 (World Health Organization, 1982) diagnostic category and stated whether suicidal ideation was a factor in the decision to admit. He recorded whether he considered the admission to be appropriate. If inappropriate, he chose an alternative management strategy from a list of options, all of which were operational. The psychiatrists were blind to the aims of the study and incomplete questionnaires were not completed at a later date to avoid bias.

The author identified medical and socio-demographic characteristics of each admission by case note review. The length of each admission was recorded. The characteristics of admissions rated inappropriate (cases) were compared with those of admissions rated appropriate (controls). Two controls were included per case, the controls being the admission rated appropriate that preceded and followed each case. When consecutive admissions were rated as inappropriate. or when inappropriate admissions were separated by a single appropriate admission, two controls preceding the first and two following the second were taken instead.

Statistical analysis included $\chi^{2}$ values, probabilities and odds ratios with $95 \%$ confidence intervals $(95 \% \mathrm{CI})$.

\section{Findings}

Two hundred and ten patients were admitted during the study period. Eighteen admissions were excluded due to the author's involvement in the admission process. One hundred and fiftyseven questionnaires were returned on the remaining 192 admissions (75\%), with $152 / 157$ $(97 \%)$ completed by senior house officers or registrars.
Thirty-eight (24\%) admissions were considered inappropriate. There was no significant difference in the proportion of cases and controls occurring out of hours (63 v. $59 \%, \chi^{2}=0.17$, $P=0.7)$.

\section{Comparison of cases and controls}

\section{Socio-demographic characteristics}

The mean age of the cases was 37.9 years (19-83 years) compared to 41.6 years (17-81 years) for the controls $(P=0.2)$. There was a significant excess of males among cases compared to controls (66 v. $43 \%, \chi^{2}=5.07, P=0.02$ ).

Cases showed higher levels of adverse sociodemographic characteristics compared to controls (Table 1). High levels of significance were reached for being single, separated, divorced or widowed as opposed to being married or cohabiting, for living alone, being of no fixed abode and for having no further education, a history of alcohol misuse, current debt or forensic involvement. High levels of significance were not reached for unemployment/retirement or a history of drug misuse.

\section{Medical characteristics}

Twenty-eight (74\%) cases received a diagnosis on admission from the F10-19 and F60-69 categories of ICD-10 (i.e. those categories primarlly concerned with substance misuse and personality disorder). This compares with $5 \quad(7 \%)$ controls. The rest of the patients in each group received a diagnosis from the F00-F09, F20-F29, F30-F39 or F40-F48 categories $(P<0.001$, odds ratio $39.8(95 \% \mathrm{CI} 12.5-127.0))$. Of note, 29 $(38 \%)$ controls received a diagnosis of schizophrenia compared to $4(11 \%)$ cases $(P<0.001)$.

Cases more commonly had a past history of parasuicide. No significant difference was found for parasuicide on admission or the expression of suicidal ideation as a factor in the decision to admit. The presence of past or family psychiatric

Table 1. Comparison of cases and controls: socio-demographic characteristics

\begin{tabular}{llcccc}
\hline Socio-demographic characteristic & $\begin{array}{l}\text { Cases } \\
(\mathbf{n = 3 8 )}\end{array}$ & $\begin{array}{l}\text { Controls } \\
(\mathbf{n = 7 6 )}\end{array}$ & $\chi^{2}$ & P value & Odds ratio (95\% Cl) \\
\hline Male & $25(66 \%)$ & $33(43 \%)$ & 5.07 & 0.02 & $2.5(1.1-5.6)$ \\
Single/separated/widowed/divorced & $34(90 \%)$ & $45(59 \%)$ & 10.91 & $<0.001$ & $6.1(2.6-14.2)$ \\
Living alone & $26(68 \%)$ & $20(26 \%)$ & 18.66 & $<0.001$ & $5.9(1.9-18.2)$ \\
Of no fixed abode & $7(18 \%)$ & $2(3 \%)$ & 8.69 & $<0.001$ & $8.4(1.6-42.5)$ \\
No further education & $31(82 \%)$ & $46(61 \%)$ & 12.24 & $<0.001$ & $2.9(1.1-7.4)$ \\
Unemployed/retired & $29(76 \%)$ & $47(62 \%)$ & 2.39 & 0.1 & $2.0(0.8-4.8)$ \\
History of alcohol misuse & $27(71 \%)$ & $8(11 \%)$ & 43.62 & $<0.001$ & $20.9(7.6-57.7)$ \\
History of drug misuse & $13(34 \%)$ & $18(24 \%)$ & 1.42 & 0.2 & $1.7(0.7-3.9)$ \\
Positive forensic history & $18(47 \%)$ & $8(11 \%)$ & 19.53 & $<0.001$ & $7.7(2.9-20.2)$ \\
History of current debt & $19(50 \%)$ & $3(4 \%)$ & 34.50 & $<0.001$ & $24.3(6.5-90.9)$ \\
\hline
\end{tabular}


Table 2. Comparison of cases and controls: medical characteristics

\begin{tabular}{lllclll}
\hline Medical characteristic & \multicolumn{2}{l}{ Cases $(n=38)$} & Controls $(n=76)$ & $\chi^{2}$ & P value Odds ratio (95\% Cl) \\
\hline Diagnosis from ICD-10 category F10-19/ & $28(74 \%)$ & $5(7 \%)$ & 55.46 & $<0.001$ & $39.8(12.5-127.0)$ \\
$\quad$ F60-69 & $26(68 \%)$ & $19(25 \%)$ & 19.99 & $<0.001$ & $6.5(2.8-15.3)$ \\
Past history of parasuicide & $16(42 \%)$ & $19(25 \%)$ & 3.48 & 0.06 & $2.2(1.0-5.0)$ \\
Suicidal ideation on admission & $10(26 \%)$ & $7(9 \%)$ & 5.84 & 0.02 & $3.5(1.2-10.2)$ \\
Parasuicide on admission & $30(79 \%)$ & $55(72 \%)$ & 0.08 & 0.8 & $1.4(0.6-3.6)$ \\
Past psychiatric history & $9(24 \%)$ & $18(24 \%)$ & 0.00 & 1.0 & $0.7(0.2-1.9)$ \\
Psychiatric family history & & &
\end{tabular}

histories in each group was approximately the same (Table 2).

\section{Outcome of admission}

The mean length of stay for cases was 7.7 days (range 1-27 days, s.d.=6.4) compared with a mean of 35.7 days (range 3-124 days, $s . d .=28.8$ ) for controls $(P<0.001)$.

\section{Alternatives to admission}

Table 3 shows the frequency of suggested alternative management plans for the 38 cases as a percentage of the total number of admissions on whom questionnaires were completed (157). All four cases of schizophrenia were considered better placed in rehabilitation beds.

\section{Comment}

The response rate was satisfactory with questionnaires completed on $75 \%$ of admissions. One-quarter of admissions were considered inappropriate by the admitting psychiatrist, a finding similar to the Nottingham study by Beck et al (1997). The proportion of cases and controls occurring out of hours was similar. Cases showed significantly higher levels of adverse socio-demographic characteristics than controls, and more commonly presented with personality disorders or substance misuse, with controls more likely to be diagnosed as suffering from severe mental illness. Cases more commonly had a past history of parasuicide, although they showed no significant excess of parasuicide or suicidal ideation at the time of admission.

Table 3. Alternative management strategies for the 38 cases

\begin{tabular}{lc}
\hline Altemative management strategy & Frequency \\
\hline Acute day ward & $13(8.3 \%)$ \\
Rehabilitation bed & $9(5.7 \%)$ \\
Urgent out-patient appointment & $2(1.3 \%)$ \\
Referral to community mental health team & $1(0.6 \%)$ \\
Referral to social services & $4(2.6 \%)$ \\
Other (e.g. medical referral, police) & $9(5.7 \%)$ \\
\hline
\end{tabular}

The mean length of stay of cases was significantly shorter than that of controls $(7.7 \mathrm{v}$. 35.7 days). Although the number of bed days used by cases seems few, this translates as a considerable usage of psychiatric beds. Thirtyeight inappropriately placed patients staying for eight days each utilise 304 bed-days over a 120day period, or in this study these patients occupied 2.5 beds per day in an 80-bed hospital.

The study has limitations. No formal definition of appropriateness was employed and ratings were made by junior rather than senior psychiatrists. The questionnaire was not formally tested for reliability or validity. However, no uniform definition of appropriateness is employed in previous studies and no rating scale for appropriateness has been developed for psychiatric use. In addition, one must consider that much of our judgement in psychiatry is subjective. The opinion of the junior psychiatrist is important since often he and the referrer act as gatekeepers to the hospital. A previous unpublished study in York demonstrated that $70 \%$ of admissions resulted from liaison between the general practitioner and junior psychiatrist, with no consultant involvement (further details available from the author upon request). This contrasts with Beck et als (1997) finding that consultants or senior registrars made the decision to admit for $75 \%$ of admissions. At the time of the study bed occupancy levels in York rarely reached $100 \%$ and perhaps with less pressure on beds, consultants were less likely to be involved in the admission process.

In the study by Creed et al (1997), a large proportion of patients with acute psychotic episodes were considered appropriate for the day ward, whereas in this study admission was considered appropriate for the vast majority of people with schizophrenia. No-one with schizophrenia was considered better placed on the day ward. Again this may reflect local policy. In York the day ward caters more for patients whose psychotic symptomatology have been stabilised by a period of in-patient treatment or for the neurotic spectrum of disorders.

Short lengths of admission have been considered to represent low levels of illness and 
inappropriate admission (Flannigan et al, 1994). If so, junior psychiatrists appear to be good judges of appropriateness of admission, since most admissions considered inappropriate lasted only a few days. However, despite considering admission to be inappropriate and having the option of other services, junior psychiatrists repeatedly admitted patients. Perhaps the ease of admission was attractive when alternative resources may be difficult and time consuming to access, or junior psychiatrists may consider particular patient groups threatening or unpredictable. Although statistical analysis did not reach significance, there is a suggestion that suicidal ideation was more common in cases than controls. It is possible that the expression of suicidal ideation acts as a form of talisman in the patient gaining admission. This along with previous suggestions warrants further study.

Perhaps the key point to emphasise is the importance of auditing the admission process. Flannigan et al (1994) suggested that clinicians need to identify those patient groups that benefit from admission with consultants offering supervision to more junior gatekeepers. The findings of this study support this view.

\section{Acknowledgement}

I thank Dr David Owens, Senior Lecturer in Psychiatry, University of Leeds for help in analysing the results.

\section{References}

Beck, T. J., Croudace, J., Singh, S., et al (1997) The Nottingham acute bed study: alternatives to acute psychiatric care. British Joumal of Psychiatry, 170. 247-252.
Creed, F.. Mbaya, P., Lancashire, S., et al (1997) Cost effectiveness of day and inpatient psychiatric treatment: results of a randomised controlled trial. British Medical Journal, 314, 1381-1385.

Flannigan, C. B., Glover, G. R., Wing, J. K., et al (1994) Inner London collaborative audit of admission in two health districts. III: Reasons for acute admission to psychiatric wards. British Journal of Psychiatry, 166. 750-759.

Gofrman, E. (1961) Asylums. New York: Doubleday.

GOLDBERG, D. \& HUXLEY, P. (1980) Mental Mlness in the Community: the Pathway to Psychiatric Care. London: Tavistock.

Hicks, N. R. (1994) Some observations on attempts to measure appropriateness of care. British Medical Journal, 309, 730-733.

HouLT, J. (1986) Community care of the acutely mentally ill. British Journal of Psychiatry, 149, 137-144.

Kent, S. \& Yellowlees, P. (1994) Psychiatric and social reasons for frequent rehospitalization. Hospital and Community Psychiatry, 45, 347-350.

Meyerson, A. T., Moss, J. Z., Belville, R., et al (1979) Influence of experience on major clinical decisions. Archives of General Psychiatry, 36, 423-427.

SCAMBLER, G. (1991) Sociology as Applied to Medicine. London: Baillière Tindall.

STEIN, L. I. \& TEST, M. A. (1980) Alternative model to mental hospital treatment. I. Conceptual model, treatment program, and clinical evaluation. Archives of General Psychiatry, 37, 392-397.

StRumwasser, I., PARANuPE, N. V., Udow, M., et al (1991) Appropriateness of psychiatric and substance abuse hospitalization: implications for payment and utilization management. Medical Care, 29 (suppl.). S77-S90.

WORLD HEALTH ORganization (1992) The Tenth Revision of the International Classification of Diseases and Related Health Problems (ICD-10). Geneva: WHO.

Peter Y. Elwood, Consultant Psychiatrist, Peter Hodgkinson Centre, County Hospital, Greetwell Road, Lincoln LN2 5UA

\title{
Solo obstetric liaison service
}

\author{
Sue Smith
}

Aims and method To set up a liaison psychiatry service for an obstetric department and review how much of the work involved in such a service can be undertaken by a senior registrar in two special interest sessions. Results It was clear there was a demand for a service for women with psychiatric problems associated with childbirth. Referrals from colleagues in general psychiatry meant that the available time was soon used up. This did therefore not allow time to develop efficient systems for referral and management or to then see the extra referrals this would have produced. The limited and temporary service was well recelved by 